\title{
INCIDENCIA Y MEDIDAS PREVENTIVAS DE HIPERTENSIÓN ARTERIAL EN DOCENTES DE INSTITUCIONES EDUCATIVAS BÁSICA, MEDIA Y SUPERIOR (UPSE) DE LOS CANTONES SALINAS Y LA LIBERTAD EN LA PROVINCIA DE SANTA ELENA. (2008 -2009)
}

\author{
MS.c Bertha Maggi Garcés \\ Profesora Investigadora Carrera de Enfermería. (UPSE) \\ berthitamaggi2@hotmail.com
}

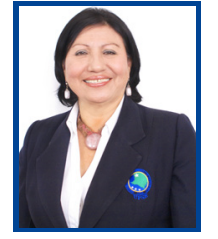

\section{RESUMEN}

La hipertensión arterial (HTA) es problema de Salud Pública a nivel mundial, la provincia de Santa Elena no es la excepción, considerando el avance espectacular de esta patología nos parece pertinente estudiar la incidencia y medidas preventivas de hipertensión arterial en nuestra población. Participan del estudio docentes de establecimientos educativos básicos y medio de los cantones Salinas y La Libertad, y docentes de la Universidad Estatal Península de Santa Elena, periodo 06/2008 - 04/2009. Los métodos: histórico lógico, deductivo, analítico comparativo y matemático. Universo y muestra 1725 docentes. Resultados, mayor incidencia de HTA en mujeres que varones, la población más afectada es entre 40 y 60 años. En el cantón La Libertad, de 419 docentes de educación media 76 (18\%) son hipertensos, de ellos 63 (83\%) son hipertensos secundarios y 13 (17\%) primarios. De 491docen 7 tes de educación básica 43 (9\%) son hipertensos, de ellos 3 (7\%) tienen HTA primaria y 40 (93\%) secundaria. En la UPSE de 285 docentes, 45 tienen HTA, de ellos, 3 (7\%) tienen HTA primaria y 42 (93\%) secundaria. En el cantón Salinas, de 366 docentes de educación básica, 32 (9\%) tienen HTA, de ellos $6(19 \%)$ son primarios y 26 (81\%) son secundarios. De 164 docentes de educación media 16 (10\%) son hipertensos, de ellos $6(37.5 \%)$ son primarios y 10 (62.5\%) secundarios. Conclusión: Hay mayor incidencia de HTA en los docentes del cantón La Libertad que en los de Salinas. Dentro de las medidas preventivas se proyectó un programa de capacitación.

\section{Palabras clave: Hipertensión Arterial, Hipertensión primaria, Hipertensión secundaria.}

\begin{abstract}
Arterial hypertension (HBP) is a world public health problem; the province of San-ta Elena is no exception, due to the spectacular progress of this disease it seems pertinent to study the incidence and preventive measures of hypertension in our population. Teachers participating in the study come from primary and secondary schools of Salinas and La Libertad, and from the State University Peninsula de Santa Elena, 06/2008-04/2009 academic period. The methods: historical, logical, deductive, comparative analytic and mathematical. Universeand sample: 1725 teachers. Results: greater incidence of HBP in women than in men. The most affected population is between 40 and 60 years old. In La Libertad, out of 419 secondary school teachers, 76 (18\%) are hypertensive, 63 of them (83\%) are secondary hypertensive and 13 (18\%) are primary hypertensive. Out of 491 primary school teachers, 49 (9\%) are hypertensive, 3 of them (7\%) have primary HBP, and 40 (7\%) secondary HBP. In UPSE, out of 285 teachers, 45 have HBP, 3 of them are primary hypertensive (6,7\%) and 42 (93.3\%) are secondary hypertensive. In Salinas, out of 366 primary school teachers, 32 (9\%) have HBP, 6 of them (19\%) are primary and 26 are secondary (81\%). Out of 164 secondary school teachers, there were 16 cases with HBP (10\%), 6 of them are primary (37.5\%) and 10 are secondary (62.5\%). Conclusion: There is more incidence of HBP in teachers from La Libertad than Salinas. A training program has been planned as a preventive measure, among others.
\end{abstract}

Keywords: Arterial hypertension, primary hypertension, secondary hypertension. 


\section{INTRODUCCIÓN}

El presente trabajo fue realizado con la colaboración de los docentes de las áreas de: Metodología de la Investigación, Morfofisiología, Enfermería básica, Salud Seguridad y Ambiente y los estudiantes del primer año, periodo académico 2008 - 2009, de la carrera de Enfermería de la Universidad Estatal Península de Santa Elena (UPSE).

De acuerdo a la Federación Mundial del Corazón, por lo menos 970 millones de personas en todo el mundo padece de hipertensión arterial, "el principal factor de riesgo de accidentes coronarios" (1). "La hipertensión estresa de tal forma que las arterias pueden obstruirse o debilitarlas. Puede llevar al estrechamiento de estos vasos sanguíneos y hacerlos más propensos a bloquearse con los coágulos o con la grasa que se acumula en sus paredes" (1). La consecuencia: Stroke, angina de pecho, insuficiencia o paros cardiacos.

Según el cardiólogo Bartolomé Finizola de la Federación Mundial del corazón dice que: “a medida que envejecemos, nuestra presión arterial sube. El problema es que cada vez más gente joven está padeciendo de hipertensión arterial, además no todos saben que la padecen y, entre quienes lo saben, no todos reciben tratamiento. Esto, definitivamente, aumenta la posibilidad de que sufran una enfermedad coronaria que los incapacite $o$ los lleve a la muerte" (1). Finizola advierte además, que la alta presión "es un enemigo silencioso".

La hipertensión arterial constituye una causa muy importante de morbimortalidad en el mundo occidental, el diagnóstico de hipertensión condiciona un estado clínico en donde la expectativa de vida del paciente se acorta y la calidad de vida se altera. La mitad de pacientes infartados son hipertensos, dos terceras partes de pacientes con stroke son hipertensos.

La hipertensión arterial es uno de los problemas médicos más comunes de la población mundial. En el mundo, 8 millones de personas mueren anualmente por hipertensión arterial (HTA), es muy serio, porque es silencioso y solamente reconocido por las lesiones de los órganos afectados. Es una molestia vascular de todo el organismo y deja marca en todos los órganos afectados: corazón, cerebro, riñones, vasos, y visión.

La hipertensión constituye una de las patologías crónicas no transmisibles, una de las de mayor prevalencia e incidencia, según Kearny el $26.4 \%$ de la población mundial adulta en el año 2000 era hipertensa, siendo el número total de hipertensos de 972 millones de pacientes de los cuales 333 millones son de los países desarrollados y 639 millones de países en vías de desarrollo y se estima que para el año 2025 se incrementaría a un 29\%.

La HTA en sus inicios es muy difícil de diagnosticar clínicamente ya que las manifestaciones que produce generalmente aparecen cuando la enfermedad ha avanzado lo suficiente como para producir daños, en los denominados "órganos blanco o diana" (2). Es un gran síndrome multifactorial que ha alcanzado notable importancia en los últimos años y a la luz de los conocimientos actuales es evidente que las cifras de presión arterial y las clasificaciones han ido variando dependiendo de los diversos estudios epidemiológicos que van surgiendo en el interés de conocer, tratar y prevenir esta entidad nosológica que aparece en la superficie como si fuera un iceberg, dejando oculto una serie de factores y fenómenos fisiopatológicos sobre lo que asientan las cifras anormales.

\section{HIPERTENSIÓN ARTERIAL (HTA) EN EL ECUADOR.}

La hipertensión arterial (HTA) es un problema de salud pública en el Ecuador y el mundo. Grave por ser silencioso en la mayoría de los casos, de ahí el término de "enemigo silencioso" (1), reconociendo esta enfermedad por las lesiones de los órganos descrito antes. Pocas personas presentan síntomas, siendo el síntoma primario la cefalea entre otros.

Según estadísticas del M.S.P. e INEC (2008), en el Ecuador existen aproximadamente 14'233.123 de habitantes, de los cuales 3'250.000 habitantes, que representa un $25 \%$ de la población sufre HTA. De este $25 \%$ de casos, 1'625.000 reciben tratamiento y de los cuales 812.000 siguen un tratamiento. En nuestro país, 3 de cada 10 ecuatorianos son hipertensos.

El Presidente de la Sociedad Ecuatoriana de Cardiología, Dr. Santiago García, explica que la alta prevalencia de 
enfermedades cardiovasculares está marcada por 6 factores de riesgo: tabaco, presión arterial alta, diabetes, colesterol alto, obesidad, factores psico-sociales (niveles de estrés) y la ausencia de factores protectores: ejercicio físico adecuado e ingesta diaria de frutas y verduras.

Según el estudio PREHTAE realizado en Quito, Cuenca y Guayaquil, 3 de cada 10 ecuatorianos adultos son hipertensos. Lamentablemente, el $60 \%$ de estos pacientes no saben que son portadores de su enfermedad, tan solo un $23 \%$ de los pacientes hipertensos recibe tratamiento y de éste, un escaso $7 \%$ alcanzan las cifras adecuadas en el control de la hipertensión arterial. En el mismo estudio, la Dra. Elsa Calero, cardióloga advierte que el peso de la enfermedad hipertensiva en el Ecuador es de tal magnitud que ocupa el primer lugar.

\section{LA HTA COMO UN PROBLEMA DE SALUD PÚBLICA EN EL ECUADOR.}

La hipertensión es un problema de salud, ubicándose en el sexto puesto con una tasa de $17.1 \%$, en los hombres, con relación a las diez principales causas de mortalidad en nuestro país según los datos del INEC del 2003, y en el quinto puesto en las mujeres con relación a los mismos datos estadísticos. Es una de las enfermedades crónicas más importantes y que más impacto tienen sobre la vida de los pacientes, después de la diabetes.

En la actualidad HTA demanda más consultas en la práctica de medicina general y en un porcentaje muy elevado a los especialistas en cardiología. Su complejidad a la luz de los últimos estudios, convier $t$ te lo que aparentemente es solo una subida anormal en valores hemodinámicos, en una enfermedad muy difícil de manejar en determinadas circunstancias. Las recientes estadísticas de HTA es la primera causa para inducir los procesos de curso clínico muy graves; la cardiopatía isquémica y la insuficiencia cardiaca congestiva, sin olvidar otras severas complicaciones como la enfermedad cardiovascular, la arterosclerosis de grandes arterias y la nefroangioesclerosis conducen a la insuficiencia renal crónica progresiva e irreversible.

\section{OBJETIVO GENERAL.}

Explicar la incidencia y las medidas preventivas de hipertensión arterial en los docentes de los es $\neg$ tablecimientos educativos básicos, nivel medio y superior (UPSE) de los cantones Salinas, La Libertad 2008-2009 para el mejoramiento de morbimortalidad y complicaciones de enfermedades cardiovascu $\neg$ lares y el conocimiento científico de los estudiantes.

\section{MATERIALES Y MÉTODOS.}

Encuestas acerca de la Hipertensión Arterial, recurso básico en nuestro estudio.

. Toma de signo vital: presión arterial, en posición sentada, con previo reposo de 10 minutos, a través de la utilización de tensiómetros aneroides y estetoscopios.

. Toma de medidas antropométricas: peso, talla, perímetro abdominal en los docentes de establecimientos básicos, medios y superiores (UPSE), de los cantones Salinas, La Libertad de la provincia de Santa Elena en el período del año lectivo 2008-2009 a través de la utilización de balanzas, y tallimetros (cintas métricas pegadas a la pared a partir de una altura de $70 \mathrm{~cm}$ y cinta métrica adicional para la medición de la cintura.

. Exámenes de laboratorio clínico: perfil lipídico, electrocardiograma en reposo.

\section{UNIVERSO Y MUESTRA.}

El universo y muestra de estudio lo constituye 1725 docentes de las instituciones educativas descritas, 910 (52,8\%) del cantón La Libertad, 530 (30,7\%) del cantón Salinas y 285 (16,5 \%) de la Universidad Estatal Península de Santa Elena (UPSE). Las unidades educativas estudiadas pertenecen a 111 escuelas, 32 colegios y 1 universidad (UPSE) de la Provincia de Santa Elena 2008 - 2009.

\section{RESULTADOS.}

Hay una mayor incidencia de HTA en los docentes del cantón La Libertad que en Salinas. En cuanto al sexo, la 
incidencia fue mayor en mujeres que en varones, de acuerdo a la edad la población más afectada está entre los 40 y 60 años.

De los 491 docentes de educación básica del cantón La Libertad la investigación reveló que existen 43 (9\%) docentes con HTA, de los cuales $3(7 \%)$ de ellos tienen HTA primaria selectiva y los 40 (93\%) restantes tienen HTA con daño renal demostrado con proteinuria y aumento de creatinina sérica, la investigación también reveló 209 docentes con sobrepeso, 77 con obesidad, 30 hombres con perímetro abdominal mayor de $102 \mathrm{~cm}$, y 171 docentes mujeres con perímetro abdominal mayor de $88 \mathrm{~cm}$.

De los docentes del cantón La Libertad, de 419 profesores de educación media 76 (18\%) son hipertensos, de los cuales 63 (83\%) docentes tiene HTA con daño renal con proteinuria y aumento de creatinina sérica y 13 (17\%) docentes HTA primaria selectiva. En cuanto al peso 133 docentes tiene sobrepeso y 71 son obesos según IMC; en cuanto a perímetro abdominal 45 docentes varones tuvieron más de $102 \mathrm{~cm}$ y 91 docentes mujeres tuvieron perímetro abdominal más de $88 \mathrm{~cm}$.

De los 366 docentes de educación básica del cantón Salinas 32 (9\%) tiene HTA, de ellos 6 (19\%) son hipertensos primarios selectivo y $26(81 \%)$ son hipertensos secundarios con daño renal - proteinuria y aumento de creatinina sérica. 137 docentes con sobrepeso y 51 obesos; perímetro abdominal 17 hombres con más de $102 \mathrm{~cm}$ y 75 mujeres con más de $88 \mathrm{~cm}$.

De los 164 docentes de educación media del cantón Salinas se encontraron $16(10 \%)$ hipertensos de los cuales $6(37.5 \%)$ son hipertensos primarios selectivo y 10 (62.5\%) son hipertensos con daño renal - proteinuria y aumento de creatinina sérica. 59 docentes tuvieron sobrepeso y 27 tienen obesidad; perímetro abdominal 19 hombres con más $102 \mathrm{~cm}, 28$ mujeres con más de $88 \mathrm{~cm}$.

En la UPSE de 285 docentes estudiados, 45 (16\%) tienen HTA de los cuales $3(7 \%)$ con HTA primaria selectiva y 42 (93\%) con HTA secundaria con daño renal - proteinuria y aumento de creatinina sérica. 27 docentes con sobrepeso, 8 con obesidad; en lo referente al perímetro abdominal 11 docentes hombres tienen más de 102 $\mathrm{cm}$ y 5 docentes mujeres tienen más de $88 \mathrm{~cm}$.

\section{DISCUSIÓN}

La hipertensión arterial (HTA) es un problema de salud pública en el Ecuador y en el Mundo. Grave por ser silencioso en la mayoría de los casos, de ahí el término de "enemigo silencioso", reconociendo esta enfermedad por las lesiones de los órganos afectados: corazón, cerebro, riñones, vasos sanguíneos y visión. Pocas personas presentan síntomas de entre los cuales el más representativo es la cefalea (dolor de cabeza en la región occipital). En nuestro país, 3 de cada 10 ecuatorianos son hipertensos. La Provincia de Santa Elena no es la excepción.

Todos los profesionales de la salud deben de saber que la HTA es una enfermedad que abarca a un porcentaje muy importante de la población, Lamentablemente, el $80 \%$ de estos pacientes no saben que son portadores de esta enfermedad, tan solo un $20 \%$ de los pacientes hipertensos recibe tratamiento. Y estos resultados se repiten en el presente estudio. Es más frecuente en mujeres que en varones. Según estudios solo la quinta parte de los afectados recibe atención médica, la pregunta es ¿cuántas personas de la población general tienen la enfermedad y no lo saben?, además se debe mencionar que la poblaาción estudiada tiene capacitación intelectual elevada.

De un total de 951 pacientes atendidos en la población general del cantón Salinas y La Libertad en año 2007 y 1er semestre del 2008 el mayor porcentaje de hipertensos fue en el cantón Salinas. En nuestro estudio el comportamiento de la hipertensión arterial en docentes fue mayor en el cantón La Libertad.

\section{CONCLUSIÓNES.}

. La hipertensión arterial es una enfermedad considerada de salud pública por el Ministerio de Salud del Ecuador.

. La Sociedad Ecuatoriana de Cardiología refiere que 3 de cada 10 ecuatorianos adultos son hipertensos. 
.Como resultado de la investigación obtuvimos lo siguiente: hay una mayor incidencia de HTA en los docentes que laboran en el cantón de La Libertad que en Salinas.

. En cuanto al sexo la incidencia fue mayor en mujeres que en varones, de acuerdo a la edad la población más afectada está entre los 40 a 60 años.

. Nuestro estudio no revela diferencia en el comportamiento de la HTA a nivel mundial.

. El trabajo contribuyó a la formación de la competencia investigativa en los estudiantes de primer año de la carrera de enfermería.

. Se capacitó en prevención de HTA primaria, secundaria, y también en estilos de vida saludable a los docentes participantes del estudio y comunidad.

\section{REFERENCIAS BIBLIOGRÁFICAS}

1. www.world-heart-federation.org Latiendo contra un enemigo.

2. Harrison. Medicina Interna. Volumen II. MaGraw Hill.2009, 1552.

\section{BIBLIOGRAFÍA.}

1. Archivos estadísticos del Departamento de Bienestar Estudiantil de los docentes de la Universidad Estatal Península de Santa Elena, 2008

2. Guyton \& Hall, Tratado de Fisiología médica, Décimo Primera Edición, Año 2007.

3. http://www.geosalud.com/hipertension/crisishipertensiva.htm.2008

4. 11http://www.abcdelasalud.net/sitio/content/view/143. 2008

5. http://html.rincondelvago.com/crisis-hipertensiva.html. 2008

6. http://www.hoy.com.ec/noticias-ecuador/tres-de-cada-10-personas-son-victimas-de-hiperten-sion-arterial-en-el-ecuador-234869-234869.html. 2008

7. http://www.monografias.com/trabajos42/problemas-de- salud/problemas-de-salud.shtml.2007.

8. Monografías de la Universidad Pedagógica Alfredo Pineda Zaldívar Habana-Cuba. 2007.

9. Revista de Thomson - Scientific\&Healthcare, Monitor Médico, Ecuador - № 40, Octubre 2007.

10. M. Cruz., Tratado de Pediatría, Océano de Ergon Nueva Edición, Año 2007

11. PLM THOMSON, Hipertensión Arterial Fascículo 5, Ecuador, Primera Edición. 2008

12. PLM THOMSON, Síndrome Metabólico, Fascículo 1, Ecuador, Primera Edición.2008 